\title{
Revisiting mycophenolate mofetil for steroid-refractory acute graft-versus-host disease: Is higher dosing effective in children?
}

Survival following allogeneic hematopoietic stem cell transplantation (HSCT) has steadily improved over time in all age groups, including children, even with increasing use of non-sibling donors $(1,2)$. Much of the improvement in outcomes is due to more effective strategies that have reduced the incidence of acute graft-versushost disease (GVHD) (3). Nonetheless, acute GVHD remains the primary cause of non-relapse mortality (NRM) after allogeneic transplantation (4). It is steroid-refractory acute GVHD, primarily involving the lower gastrointestinal (GI) tract, that accounts for the vast majority of NRM (5). Unfortunately, there are no proven treatments for steroid-refractory acute GVHD despite over $40 \mathrm{yr}$ of clinical trials. It is in this context that Inagaki et al. (6) studied mycophenolate mofetil (MMF) as a treatment for steroidrefractory acute GVHD as reported in this issue of Pediatric Transplantation.

Inagaki et al. analyzed the effectiveness of oral MMF in 14 children with steroid-refractory acute GVHD that had not responded to at least two lines of treatment before beginning MMF. They report an impressive $83 \%$ response rate at eight wk after initiation, which included an equally high response rate in patients with GI GVHD. The vast majority of patients remained alive, in remission, and off all immunosuppression at a median of almost three yr of follow-up. Although second-line therapies are often associated with significant toxicities (7), MMF was very well tolerated in this study. The authors attribute the success of their treatment to the high starting dose $(>40 \mathrm{mg} / \mathrm{kg} /$ day $)$ and further dose escalation due to poor initial dose response, an approach supported by data showing a strong dose-response relationship between MMF and acute GVHD incidence and response to treatment $(8,9)$.
Although the results from this study are promising, they are far from definitive, especially in light of the small numbers of patients studied. Only oral MMF was used despite the high incidence of voluminous diarrhea, and no pharmacokinetic studies were performed, so we cannot be sure whether the high dosing actually led to higher blood concentrations of active drug. Also, several patients responded despite cessation of steroid therapy prior to initiating MMF, a strategy that raises questions as to how steroid-refractory the GVHD was in some patients. We also do not know whether some of the responses observed were from ongoing or delayed response to second- or higher line therapy already administered. In addition, we need to reconcile the results of this study with the large, multicenter, phase III randomized, controlled trial that failed to show any benefit from MMF compared to placebo when used in combination with steroids to treat newly diagnosed acute GVHD (10).

Although Inagaki et al. observed excellent responses to MMF in their patients, we do not know whether these results will be reproducible in future trials. If we can identify patients whose GVHD will be resistant to frontline therapy before treatment failure has occurred, we may be able to design better clinical trials in the future. There has been intensive effort recently to develop staging systems that will help us categorize patients for risk of treatment failure when they are diagnosed with GVHD. MacMillan et al. (11) developed a clinical risk score for acute GVHD that predicts response to treatment, NRM, and survival based on GVHD target organ involvement and clinical severity when treatment is begun. Patients with isolated, but severe skin, GI, or liver involvement, and most combinations of multi-organ GVHD, are classified as high risk and comprise approximately 
$15 \%$ of all GVHD cases treated. These patients experience low response rates to initial steroid therapy $(43 \%$ complete or partial response rate by day 28 of treatment), high six-month NRM $(44 \%)$, and poor six-month survival $(53 \%)$. Plasma concentrations of GVHD biomarkers, alone or in combination, have also been extensively studied for their ability to predict response to treatment, NRM, and survival (12-14). The recently published Ann Arbor GVHD scoring system defined and validated three risk strata based entirely on the plasma concentrations of three GVHD biomarkers, TNFR1, REG3 $\alpha$, and ST2 measured at GVHD diagnosis in a training cohort and two independent validation sets (5). This study included patients whose initial treatment did not include systemic immunosuppression such as those who presented with limited GVHD skin rashes. Patients with high-risk (Ann Arbor 3) GVHD, who comprise $22 \%$ of all GVHD cases, experienced low response rates to initial therapy $(46 \%)$, high 12-month NRM $(49 \%)$, and low 12 -month survival $(45 \%)$. Either scoring system, or possibly a combination, represents a new opportunity to tailor primary therapy according to the risk of steroid-refractory GVHD before it develops, when it is more likely to respond.

In summary, Inagaki et al. have revived interest in MMF as a GVHD treatment, at least for children with steroid-refractory GVHD. New GVHD scoring systems that can be applied at diagnosis may lead to better designed clinical trials in the future that focus on improving outcomes for high-risk patients. For example, it is possible that agents that previously failed to show benefit for initial GVHD treatment, such as MMF, may yet prove efficacious when tested only in high-risk patients, especially if dosing is determined based on pharmacokinetics. If this is indeed the case, it could help reconcile the current contradictory results between the present positive study (6) and the negative randomized controlled trial (10).

\section{Conflict of interest}

The author declares no conflict of interest.

John E. Levine

Pediatric Blood and Marrow Transplantation Program, University of Michigan, 1500 E. Medical Center Drive, Ann Arbor, MI 48109, USA

E-mail: jelevine@umich.edu

\section{References}

1. Hahn T, McCarthy PL $J_{R}$, Hassebroek A, et al. Significant improvement in survival after allogeneic hematopoietic cell transplantation during a period of significantly increased use, older recipient age, and use of unrelated donors. J Clin Oncol 2013: 31: 2437-2449.

2. MacMillan ML, Davies SM, Nelson GO, et al. Twenty years of unrelated donor bone marrow transplantation for pediatric acute leukemia facilitated by the National Marrow Donor Program. Biol Blood Marrow Transplant 2008: 14 (Suppl): 16-22.

3. Davies SM, Wang D, WANG T, et al. Recent decrease in acute graft-versus-host disease in children with leukemia receiving unrelated donor bone marrow transplants. Biol Blood Marrow Transplant 2009: 15: 360-366.

4. Ferrara JL, Levine Je, Reddy P, Holler E. Graft-versushost disease. Lancet 2009: 373: 1550-1561.

5. Levine Je, Braun TM, Harris AC, et al. A prognostic score for acute graft-versus-host disease based on biomarkers: A multicenter study. Lancet Haematol 2015: 2: e21-e29.

6. Inagaki J, Kodama Y, Fukano R, Noguchi M, Okamura J. Mycophenolate mofetil for treatment of steroid-refractory acute graft-versus-host disease after pediatric hematopoietic stem cell transplantation. Pediatr Transplant 2015: 19.

7. Martin PJ, Rizzo JD, Wingard JR, et al. First- and secondline systemic treatment of acute graft-versus-host disease: Recommendations of the American Society of Blood and Marrow Transplantation. Biol Blood Marrow Transplant 2012: 18: $1150-1163$

8. Harnicar S, Ponce DM, Hilden P, et al. Intensified mycophenolate mofetil dosing and higher mycophenolic acid trough levels reduce severe acute graft-versus-host disease after double-unit cord blood transplantation. Biol Blood Marrow Transplant 2015: 21: 920-925.

9. JaCOBSON PA, HuAng J, Wu J, et al. Mycophenolate pharmacokinetics and association with response to acute graft-versushost disease treatment from the Blood and Marrow Transplant Clinical Trials Network. Biol Blood Marrow Transplant 2010: 16: 421-429.

10. Bolaños-Meade J, Logan BR, Alousi AM, et al. Phase 3 clinical trial of steroids/mycophenolate mofetil vs steroids/placebo as therapy for acute GVHD: BMT CTN 0802. Blood 2014: 124: 3221-3227.

11. MacMillan ML, Robin M, Harris AC, et al. A refined risk score for acute graft-versus-host disease that predicts response to initial therapy, survival, and transplant-related mortality. Biol Blood Marrow Transplant 2015: 21: 761-767.

12. Ferrara Jl, Harris AC, Greenson JK, et al. Regenerating islet-derived 3-alpha is a biomarker of gastrointestinal graftversus-host disease. Blood 2011: 118: 6702-6708.

13. XIAO B, WANG Y, LI W, et al. Plasma microRNA signature as a noninvasive biomarker for acute graft-versus-host disease. Blood 2013: 122: 3365-3375.

14. Vander Lugt MT, Braun TM, Hanash S, et al. ST2 as a marker for risk of therapy-resistant graft-versus-host disease and death. N Engl J Med 2013: 369: 529-539. 